\title{
The influence of information structuring and health literacy on recall and satisfaction in a simulated discharge communication
}

\author{
Victoria Siegrist $^{\mathrm{a}, \mathrm{b}}$, Wolf Langewitz ${ }^{\mathrm{c}, *}$, Rui Mata ${ }^{\mathrm{b}}$, Dominik Maiori ${ }^{\mathrm{b}, \mathrm{d}}$, Ralph Hertwig ${ }^{\mathrm{e}}$, \\ Roland Bingisser ${ }^{\mathrm{a}}$ \\ a Emergency Department, University Hospital Basel, Basel, Switzerland \\ ${ }^{\mathrm{b}}$ Center for Cognitive and Decision Sciences, University of Basel, Basel, Switzerland \\ ${ }^{\mathrm{c}}$ Department of Psychosomatic Medicine, University Hospital Basel, Basel, Switzerland \\ d Department of Digital Film Production, SAE Institute Zurich, Zurich, Switzerland \\ e Center for Adaptive Rationality, Max Planck Institute for Human Development, Berlin, Germany
}

\section{A R T I C L E I N F O}

\section{Article history:}

Received 22 January 2018

Received in revised form 30 July 2018

Accepted 5 August 2018

\section{Keywords:}

Health literacy

Information structuring

Discharge communication

Physician-patient communication

Information recall

Emergency medicine

Book metaphor

\begin{abstract}
A B S T R A C T
Objective: We investigated the effects of information structuring and its potential interaction with preexisting medical knowledge on recall in a simulated discharge communication.

Methods: 127 proxy-patients (i.e. students) were randomly assigned to one of four conditions. Video vignettes provided identical information, differing in means of information structuring only: The natural conversation (NC) condition was not explicitly structured whereas the structure (S) condition presented information organised by chapter headings. The book metaphor (BM) and the post organizer (PO) conditions also presented structured information but in addition included a synopsis, either at the beginning or at the end of discharge communication, respectively. Proxy-patients' recall, perception of quality and pre-existing medical knowledge were assessed.

Results: Information structuring (conditions: S, BM, PO) did not increase recall in comparison to NC, but pre-existing medical knowledge improved recall $(p<.01)$. An interaction between medical knowledge and recall in the BM condition was found $(p=.02)$. In comparison to the NC, proxy-patients in all information structuring conditions more strongly recommended the physician $(p<.001)$.

Conclusions: Structured discharge communication complemented by the BM is beneficial in individuals with lower pre-existing medical knowledge.

Practice implications: The lower pre-existing medical knowledge, the more recipients will profit from information structuring with the BM.
\end{abstract}

(C) 2018 Published by Elsevier B.V.

\section{Introduction}

Discharge from the emergency department is a crucial event often accompanied by stress and vulnerability. Patients' understanding of key aspects of post-discharge care is generally described as poor $[1,2]$ yet strategies for fostering the transition are still little understood [3], with some authors advocating a 'resuscitation of physician-patient communication' [4]. For instance, recent evidence suggests no benefit of electronic tools in discharge communication on readmission rates, adverse events, or mortality [5,6]. Patients who cannot recall or comprehend discharge plans are vulnerable to risks of medical

\footnotetext{
* Corresponding author at: Petersgraben 2, 4031, Basel, Switzerland.

E-mail address: wolf.langewitz@usb.ch (W. Langewitz).
}

error, adverse events or failure to seek further help from healthcare providers [7]. Consequently, the ability to recall information provided during discharge is an indispensable condition for patients' informed decisions and well-being. Unfortunately, research suggests that patients' recall and comprehension is severely limited and can negatively impact their health-related choices [8,9]. As a result of ever increasing time constraints, patient-centred communication is severely under pressure. Scientific advances have accelerated diagnostic pathways and rendered possible 'fast-track' emergency care, such as the rapid exclusion of myocardial infarction [10]. Therefore, an expeditious diagnostic process needs to be matched by timely and efficient discharge communication.

Oral information structuring has been shown to be a powerful tool for boosting memory and recall in educational settings [11], and pre-existing knowledge structures have been demonstrated to 
foster the processing and future recall of new information in standard memory experiments [12]. Less is known about the role of information structuring and pre-existing knowledge in the medical domain and, specifically, in the context of discharge communication. Information structuring has been found to boost recall in simulated emergency discharge communications [13]. An early landmark study suggested providing patients with an initial and brief synopsis prior to the provision of the full set of detailed information [14]. Analogous to the table of contents of a book -we refer to this method as the 'book metaphor' (BM) [13,15]- recall of medical information may be improved. The BM can be thought of as a specific implementation of an 'advance organizer' (AO) successfully used in educational contexts. Similarly, 'post organizers' (PO), synopses provided at the final stage of the process of information provision, may also foster patients' recall performance $[16,17]$. Crucially, there is some indication that patients with lower health literacy [18] may especially benefit from information structuring [15], because such a 'structure' provides a scheme that helps to represent and store information. Individuals with higher health literacy are, at least partially, in possession of such a scheme that might help to encode and retrieve information. So far, few studies have considered the possibility that information structuring can facilitate the process of conveying information to individuals with different levels of health literacy.

Therefore, we examined the impact of information structuring in the context of discharge communication and its potential interaction with pre-existing medical knowledge. Our first objective was to assess the effect of information structuring on recall performance as well as any potential interaction with individuals' pre-existing medical knowledge. In addition, we tested for a possible trade-off between recall and proxy-patients' perceptions of quality. Such a trade-off could mean that any benefits of structured communication come at the expense of lower satisfaction with the physician relative to a less structured but more natural conversation style.

\section{Methods}

\subsection{Participants}

A convenience sample of students were recruited as proxypatients via an online platform of the Department of Psychology, University of Basel, Switzerland. The study description spelled out inclusion criteria: less than 30 years of age, no hearing impairment, and native German speaker. Students were reimbursed for their participation in terms of credit points or 7.50 Swiss Francs. The study was approved by the institutional review board of the Department of Psychology (015-16-1).

\subsection{Study material}

We designed four highly standardised video clips featuring a discharge communication in the emergency department (ED) to individuals with varying degrees of pre-existing medical knowledge. The three information-structuring videos simulated a discharge event involving the use of an $\mathrm{AO}$, a PO, and structured communication without an $\mathrm{AO}$ or a PO (S). These conditions were then compared to an unstructured communication event emulating a physician's natural conversational style (NC) (Fig. 1). The four video clips depicted an ED physician, recorded from a first person's view. The physician was oriented toward an imaginary patient (the viewer). The patient had presented with chest pain to the ED and was to be discharged after a myocardial infarction could be ruled out. Further work-up was warranted. The study material (i.e., video vignettes) is available online at the following Open Science Framework workspace: osf.io/w5bpa.

In the NC condition, the same information as in the other conditions was presented but without any explicit structure. Segments were presented in a natural order, as judged by emergency physicians practising such routines on a daily basis. In the $S$ condition, the same segments were presented but now in the following well-defined order with additional chapter headings according to the mnemonic acronym InFARcT: Information on diagnosis (In), Follow-up suggestions (F), Advice on self-care (A), Red flags (R), and complete Treatment (cT) [19]. In the BM condition, an AO preceded the same sequence of segments: 'We will now talk about the following five topics.... In the PO condition, the sequence of segments was concluded with a PO: 'We have just talked about the following five topics ....'.

All videos featured an emergency physician who presented the identical set of 42 items of information (see Appendix A). The videos employed the same footage and differed only by means of information structuring (i.e., order of the information, chapter headings, and the organizer). Each of the different video segments consisted of one to six items of information. The individual segments were interrupted by black screens of $0.8 \mathrm{~s}$ duration in order to permit the organisation of the segments in the defined orders. The duration of the video clips was 3:52 min (NC condition), 4:23 min (S condition) and 4:46 min (BM condition and PO condition). The differences in duration can be fully explained by the means of information structuring. The additional provision of chapter headings took $31 \mathrm{~s}$ and the provision of a synopsis took $24 \mathrm{~s}$. Although there was a time difference of up to $55 \mathrm{~s}$ between conditions, the additional elements of information structuring did not contain any additional factual information.

To assess participants pre-existing medical knowledge, a multiple-choice test was used. It consisted of twelve questions

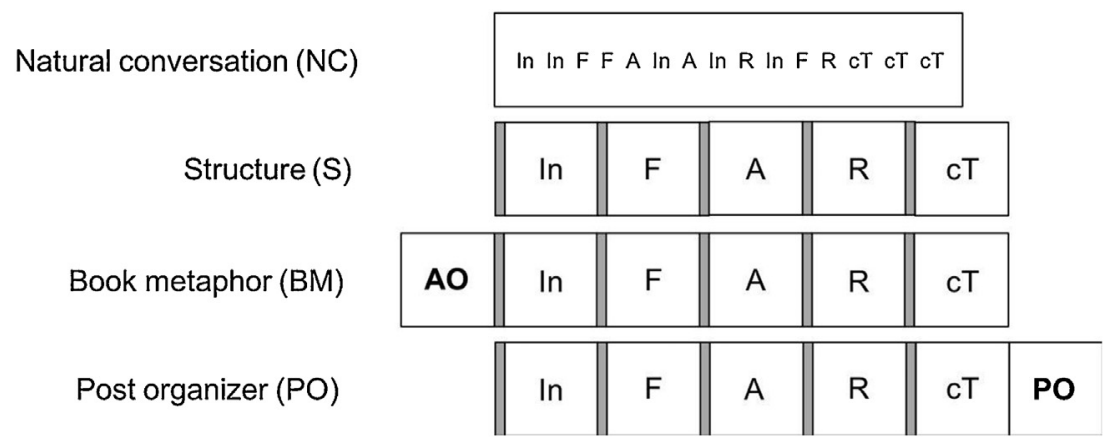

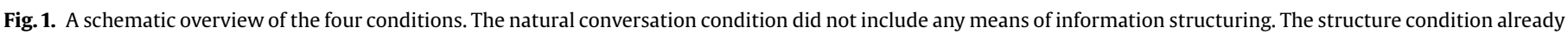

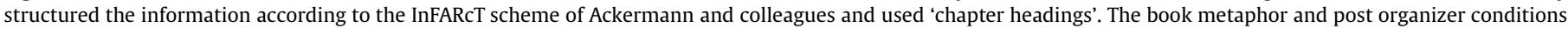

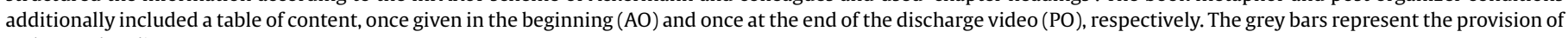
a chapter heading. 
of which each had four answer options, of which only one was correct (see Appendix A). The test was validated in a preliminary study, using undergraduate year 1 psychology students and undergraduate year 1 and year 3 medical students. We chose this sample, assuming that psychology students have the lowest preexisting medical knowledge, followed by medical students in their first year and medical students in their third year. To examine the validity of the test, undergraduate year 1 psychology students (P1), undergraduate year 1 medical students (M1) and year 3 medical students (M3) were recruited. Each group consisted of 30 participants. P1's mean age was 21.4 years $(S D=1.7)$, while M1's and M3's mean age was 20.7 years $(S D=2.1)$ and 22.2 years $(S D=1.7)$, respectively. $83.3 \%$ of the P1 group and $66.7 \%$ of both medical student groups (i.e., M1 and M3) were women. The test showed a mean inter-item-correlation of .1 and a Cronbach's $\alpha$ of .7. Computing a logistic regression, with number of items correct as the dependent and group as the independent variable, the test succeeded in distinguishing between the three student groups $(p<$ .001).

\subsection{Study protocol}

In order to standardize the study (e.g. setting and procedure), proxy-patients were given an appointment for their participation at the laboratory of the psychology department of the University of Basel, Switzerland. Before the study began, proxy-patients gave their informed consent. After signing informed consent, they were randomly assigned to one of the four conditions. They were instructed to adopt a patient's perspective and to evaluate the discharge communication depicted in the video. After the presentation of the discharge video, individually on computer screens, proxy-patients rated the quality of discharge communication on four dimensions, using visual analogue scales (VAS). Each VAS ranged from 0 to 10 and the dimensions were (1) how easy to understand was the communication?, (2) how structured was the communication?, (3) how informative was the communication?, and (4) how strongly would you recommend the physician to family and friends?. After their evaluation of the quality of discharge communication, proxy-patients were asked to write down all the information that they could remember from discharge. The minimum recall period was a time-slot of $5 \mathrm{~min}$. No proxy-patient extended the recall period beyond these $5 \mathrm{~min}$. Furthermore, a questionnaire probed proxy-patients' pre-existing medical knowledge (see Appendix A). Finally, demographic data (i.e., age, sex, and occupation) and control variables (i.e., native language and comments to the researcher) were recorded.

\subsection{Transcript coding}

The occurrence of an element from the 42 items of information was coded by comparing proxy-patients' entries into their memory protocol with the information given (see Appendix A). We examined intercoder reliability between two raters, using a subset of 40 proxy-patients. Intercoder reliability refers to the extent to which two independent coders agree on the coding of items in question, assuming the same coding scheme [20]. The intraclass correlation (ICC) for the total amount of items recalled was very high $($ ICC $=0.95)$.

\subsection{Statistical analyses}

All analyses were performed using the software $\mathrm{R}$ (Version 3.4.1). We calculated Pearson correlation coefficients between the number of items recalled and the medical knowledge score to quantify the degree of association between these two variables. Additionally, a multiple regression analysis with number of items recalled as the dependent variable, and degree of pre-existing medical knowledge and the factor condition as independent variables was calculated. The NC condition was taken as the comparison condition. Pre-existing medical knowledge and condition were entered as an interaction into the model. Preexisting medical knowledge was centered to the mean knowledge of proxy-patients in order to make the estimates easier to interpret. Finally, we computed linear regressions for all four measurements of proxy-patients' subjective ratings of quality, with condition as independent variable. A $p$-value of $p<.05$ was considered statistically significant. The data analysis script, data, and the coding scheme are available online at the following Open Science Framework workspace: osf.io/w5bpa.

\section{Results}

\subsection{Participants}

A total of 133 proxy-patients were recruited of which 127 were used for data analysis. We excluded five proxy-patients for the following reasons: software failure during data collection $(n=2)$; medical diploma $(n=2)$ and hearing impairment $(n=1)$. Additionally, one proxy-patient was excluded due to a very strong preference for alternative medicine, prompting exceedingly low ratings in quality $(n=1)$. This proxy-patient approached the experimenter after participation and stated that the ratings of quality only reflect her preference for alternative medicine, and not the actual satisfaction with the physician or the discharge communication. Proxy-patients' age ranged from 18 to 29 years with a mean age of 21.6 years $(S D=2.6) ; 82.7 \%$ were female and $85 \%$ were undergraduate psychology students.

\subsection{Primary outcome: information recall and pre-existing medical knowledge}

In line with the findings from Ackermann et al. [15], a Pearson correlation analysis indicated a significant positive association between pre-existing medical knowledge and recall across all conditions $(r(125)=.28, p=.002)$. Analysing the correlation separately for the four different conditions, however, we found significant associations between recall and pre-existing medical knowledge in the NC and the S conditions but not in the PO and BM conditions (see Table 1). As can be seen in Fig. 2, prior medical knowledge is positively associated with information recall in most conditions, with the smallest correlation in the BM condition $(\mathrm{r}(30)$ $=-.04, p=.85$ ).

We conducted a multiple regression analysis (see Section 2.5) to test for the role of information structuring in improving recall relative to a natural conversation style and to find out whether benefits of information structuring are associated with individuals' pre-existing medical knowledge (see Table 2). We found that no condition was significantly related to increased recall relative to the NC condition; however, we observed a significant interaction between pre-existing medical knowledge and information recall in the BM condition, suggesting that the relation between medical

Table 1

Correlations between medical knowledge and recall by condition.

\begin{tabular}{lll}
\hline Condition & $r$ & $p$-value \\
\hline Natural conversation (NC) & .57 & $<.001^{* * *}$ \\
Structure (S) & .38 & $.037^{*}$ \\
Book metaphor (BM) & -.04 & .85 \\
Post organizer (PO) & .24 & .18 \\
\hline
\end{tabular}

Note. The correlation coefficient $r$ was computed with a Pearson correlation. ${ }^{*} p<.05$. ${ }^{* *} p<.01$. ${ }^{* * *} \mathrm{p}<.001$. 


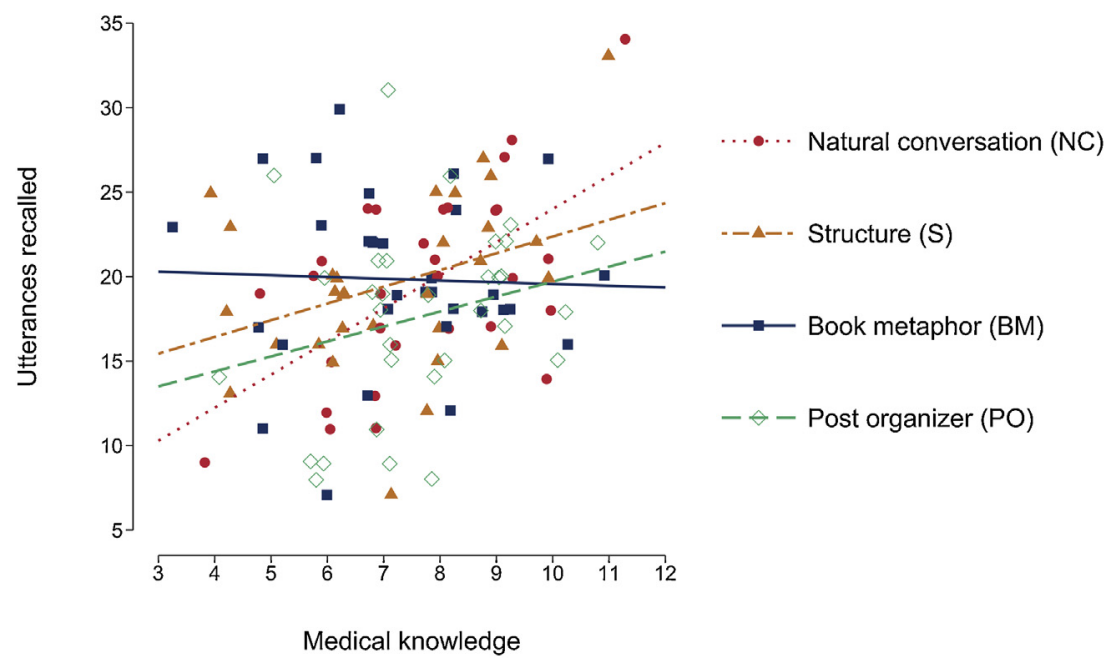

Fig. 2. Scatterplot with regression lines showing the relationship between medical knowledge scores and recall of discharge information clustered by condition.

Table 2

Results from multiple regression analysis with the dependent variable recall.

\begin{tabular}{lllll}
\hline & Estimate & Std. Error & t-value & $p$-value \\
\hline Intercept & 19.02 & .92 & 20.60 & $<.001^{* * *}$ \\
Structure (S) & .42 & 1.32 & .32 & 0.75 \\
Book metaphor (BM) & .81 & 1.30 & .63 & 0.53 \\
Post organizer (PO) & -1.57 & 1.31 & -1.20 & 0.23 \\
Medical knowledge & 3.30 & .99 & 3.33 & $.001^{* *}$ \\
Structure:medical knowledge & -1.38 & 1.30 & -1.07 & .29 \\
Book metaphor:medical knowledge & -3.48 & 1.35 & -2.58 & $.01^{*}$ \\
Post organizer:medical knowledge & -1.81 & 1.42 & -1.27 & .21 \\
\hline
\end{tabular}

Note. In the regression analysis pre-existing medical knowledge was centred to the mean knowledge of proxy-patients. This measure was taken to make the estimates easier to interpret. Our analysis shows an $R^{2}$ of 0.16 and an adjusted $R^{2}$ of 0.11 . * $p<.05 .{ }^{* *} p<.01$. ${ }^{* * *} \mathrm{p}<.001$.

knowledge and information recall was different in the BM relative to the remaining conditions.

\subsection{Secondary outcome: ratings of quality}

Linear regressions were used to examine the relationship between proxy-patients' ratings of quality and communication condition. Confirming the validity of our experimental conditions, proxy-patients' rated discharge communication as more structured in the three structured conditions relative to the NC condition $(F(3,123)=18.62, p<.001)$ : S condition $(p<.001)$, BM condition $(p<.001)$ and PO condition $(p<.001)$. Additionally, proxypatients more strongly recommended the physician to family and friends in all three structured conditions relative to the NC condition $(\mathrm{F}(3,123)=6.43, p<.001)$ : $\mathrm{S}$ condition $(p<.001)$, BM condition $(p<.001)$ and PO condition $(p<.001)$. In contrast, there was no significant difference on the dimensions of comprehensibility $(F(3,123)=1.15, p=.33)$, and informativeness $(F(3,123)=1.43$, $p=.24$ ) between conditions. Mean ratings and SD by condition can be found in Table 3 .

\section{Discussion and conclusion}

\subsection{Discussion}

Effective discharge communication is crucial to ensure the successful transition from hospital to ambulatory care, yet past research suggests that comprehension and recall of such information is poor [21-23]. We aimed to foster recall of discharge communication by examining the combination of information structuring and the use of advance organizers or post organizers; that is, synopses previewing or retrospectively summarising information. More specifically, we studied how such communication methods can improve recall performance in individuals with varying degree of pre-existing medical knowledge.

Our study found some support for the advantage of information structuring for information recall and patients' subjective ratings of comprehension. Importantly, the results suggest that information structuring, particularly with the book metaphor, has a beneficial role and can be a powerful tool to eliminate the disadvantage of low pre-existing medical knowledge in recall of new information. Our results thus may represent a first small step towards designing effective communication strategies that can help patients with diverse backgrounds and contribute to mitigating health inequalities.

The mnemonic benefits of information structuring, relative to a natural conversation style is not new [11,13]. However, past work

Table 3

Proxy-patients' subjective ratings of quality by condition.

\begin{tabular}{|c|c|c|c|c|c|c|c|c|}
\hline \multirow[b]{2}{*}{ Condition } & \multicolumn{2}{|c|}{ Structuredness } & \multicolumn{2}{|c|}{ Recommendation } & \multicolumn{2}{|c|}{ Comprehensibility } & \multicolumn{2}{|c|}{ Informativeness } \\
\hline & Mean & $S D$ & Mean & $S D$ & Mean & $S D$ & Mean & $S D$ \\
\hline Natural conversation (NC) & 6.4 & 2.3 & 6.6 & 2.4 & 8.8 & 1.6 & 7.5 & 1.9 \\
\hline Structure $(\mathrm{S})$ & 9.0 & 1.1 & 8.5 & 1.3 & 9.2 & 0.9 & 8.2 & 1.2 \\
\hline Book metaphor (BM) & 8.8 & 1.2 & 7.9 & 1.7 & 9.2 & 1.1 & 8.0 & 1.9 \\
\hline Post organizer (PO) & 8.7 & 1.7 & 8.0 & 1.8 & 9.2 & 0.9 & 7.6 & 1.4 \\
\hline
\end{tabular}

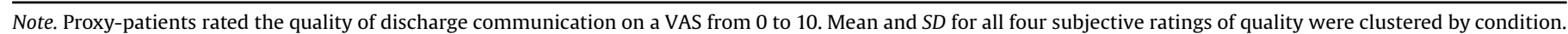

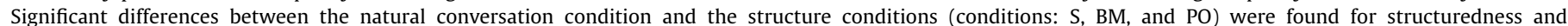
recommendation. No significant differences were found for comprehensibility and informativeness. 
has not evaluated the independent contributions of structure and the organising synopsis of an AO vs PO [13]. In the present study, these elements were analysed separately, we thus were able to evaluate whether certain elements of explicit information structuring are especially helpful to either improve recall or to mitigate the influence of pre-existing medical knowledge on recall. Our results suggest that an $\mathrm{AO}$ ("In the following I will talk about five different aspects: first ... . . ") may be particularly helpful for individuals with little prior medical knowledge whereas such an effect could not be demonstrated with a résumé at the end of providing information ("I have been talking about five different aspects: first...."). Importantly, these mnemonic benefits of structure do not need to be traded against other important quality dimensions of patient-physician communication: to the contrary, proxy-patients were more likely to recommend the physician to family and friends if discharge communication was explicitly structured.

One of our limitations is that we used video-taped rather than face to face interactions; therefore, we cannot be sure whether in real life we would have found similar results. However, we wanted to ensure strict standardisation of the content of discharge information. In a natural setting and given a sensitive patientcentred professional, patients have a chance to contribute to the speed of delivery of information by non-verbal cues like nodding, gaze direction, etc. or by verbal input, e.g. by asking questions. Although such adaptive and finely tuned communication strategies are, in principle, possible, the ample evidence for poor discharge communication suggests that in reality they are not widely used.

Admittedly, the knowledge about signs, symptoms, and risk factors of myocardial infarction (as tested here) represents only a small slice of health literacy [18]. Yet, we found a clear relationship between pre-existing medical knowledge and recall performance, most pronounced in the NC condition, and almost eliminated in the book metaphor condition. In order to fully appreciate this link, it is important to note that it is likely that general health literacy is above average in the present sample of university students relative to the population of emergency department patients. In other words, our study may, if anything, underestimate the link between recall performance and pre-existing medical knowledge. The book metaphor appears to mitigate or even eliminate this link. This is obviously important for the average emergency patient, who is expected to retain dozens of often new pieces of information when being discharged [24]. Past studies have shown that standardised [25] or simplified [26] discharge information may increase comprehension and recall. Yet, the crucial link between health literacy and memory performance has previously received little attention [15].

A further limitation is that university students are not representative for the typical patient presenting to an ED with symptoms of chest pain: Using students as proxy-patients came with a gender imbalance in favour of women. Besides, patients are likely to be older and less educated and certainly less used to memorising new information. Note, however, that our results do not seem to be the product of ceiling effects; there was still a large variance of the percentage of items recalled: Only $37 \%$ of the students remembered more than half of the items provided, the best performing students recalled around $80 \%$ of all items. Regardless, the students' age $[27,28]$ and routine of memorising information is an advantage. In addition, students did not suffer from the potentially high levels of stress or anxiety emergency patients experience at work-up and discharge [29]. Taken together, one may therefore expect that our results overestimate the recall performance of patients in emergency care and, by extension, perhaps underestimate the benefit of a structured discharge communication using an AO.

\subsection{Conclusion}

Structuring of discharge information and the use of the book metaphor appears to be a relatively simple and cost-effective method of compensating for the heterogeneity in pre-existing medical knowledge. This is a relevant advantage because the patients' ability to remember discharge information is linked to pre-existing medical knowledge. Before these findings are implemented into student education and physician training, however, the data presented in a student population need to be replicated in a prospective randomised study with patients discharged from the ED.

\subsection{Practise implications}

In daily practise, it is difficult to assess pre-existing medical knowledge. Therefore, the usefulness of a communication tool should be independent of the patients' medical knowledge. Our data supports the use of the book metaphor due to the fact that especially in limited pre-existing knowledge recall of information can be improved.

For teaching purposes, we coined the term 'book metaphor' instead of advance organizer because it readily elicits a conception of the explicit structure featuring a table of content, chapter headings, and text - analogous to the structure of a textbook.

\section{Contributors}

All authors contributed to the study and have approved this version of the manuscript for publication.

\section{Funding}

This work was supportedby the Swiss National Foundation [grant number [CR31I3_159841/1].

\section{Ethics approval}

University of Basel Department of Psychology Institutional Review Board (015-16-1).

\section{Acknowledgements}

We thank Markus Steiner for implementing the study, Leona Knusel for data collection, Valentina Steffen for coding, and Laura Wiles for editing the manuscript.

\section{Appendix A}

\section{A.1 Assessment of pre-existing medical knowledge}

The following 12 multiple choice questions were used to assess participants' pre-existing medical knowledge. Participants were instructed to choose only one answer option. The correct answers are indicated in bold.

1) Hypertension is dangerous because

a) It can lead to diabetes mellitus

b) It can lead to cancer

c) It can lead to stroke

d) It may damage the lungs

2) A myocardial infarction (heart attack) is

a) An occlusion of a cardiac artery

b) An occlusion of a cardiac vein

c) A tear in the heart muscle

d) A tear in a heart valve

3) A normal value of blood pressure is: 

a) $200 / 60 \mathrm{mmHg}$
b) $170 / 60 \mathrm{mmHg}$
c) $160 / 60 \mathrm{mmHg}$
d) $120 / 60 \mathrm{mmHg}$

4) Which of the following symptoms is normally not caused by a heart attack?

a) Pain in the left shoulder

b) Pain in the jaw

c) Headaches

d) Upper abdominal pain

5) Which condition does not increase the risk for a heart attack?

a) Nicotine consumption

b) Chronic bronchitis

c) Elevated cholesterol levels

d) Hypertension

6) Smoking increases the risk of:

a) Calcification of blood vessels

b) Diabetes mellitus

c) Glaucoma

d) Liver damage

7) Myocardial infarction is:

a) A suddenly occurring irregularity of the rhythm of the heart, leading to severe pain.

b) A slowly occurring narrowing of the coronary arteries

c) A suddenly occurring weakness of the heart muscle

d) Death of the heart muscle due to lack of oxygen

8) Angina pectoris refers to:

a) Shortness of breath combined with galloping heart rhythm

b) Pain due to heart overload

c) Pain due to momentarily inadequate supply of oxygen to the heart muscle

d) Galloping heart rhythm due to momentarily inadequate supply of oxygen to the heart muscle

9) What is a risk factor for diseases of the cardiovascular system:

a) High level of physical exertion (heavy labour, high-performance sport)

\section{b) Diabetes}

c) Frequent viral infections

d) Electromagnetic radiation (for example, mobile phones)

10) Typical pain during a heart attack:

a) Becomes stronger with deep breathing

b) Radiates to the left arm

c) Comes with sudden movement

d) Radiates from the left chest area to the right chest area

11) A cardiac-catheterisation procedure is:

a) An ultrasound of the coronary arteries

b) A computed-tomography scan of the heart

c) An ultrasound of the heart

d) A radiographic investigation of the coronary arteries

12) Typical cardiac pain becomes worse with:

a) Rapid breathing

a) Emotional stress

c) Physical effort

d) Raising of the arms

\section{A2. Utterances conveyed during discharge communication}

\section{Item}

Patient can go home

Right now it is no myocardial infarction

Patient was reassured (you were right to come here")

Presumptive diagnosis of Angina Pectoris

Explanation of Angina Pectoris (narrowing of cardiac blood vessels“)

Narrowing of cardiac vessels means problems with oxygen supply

Narrowing of cardiac vessels is painful under streneous exercise
(Continued)

Item
Myocardial scintigraphy will be performed
Myocardial scintigraphy clarifies the extent of narrowing
Myocardial scintigraphy will be performed next week
Myocardial scintigraphy will be done here in hospital
Information on time and location of the test will be sent by post
Prior to myocardial scintigraphy
$\ldots$ no coffee
$\ldots$ no tea
.. no chocolate
Patient was told try to abstain from smoking
Patient was told to avoid physical stress
Patient was told to come back to the ED
... if he was dyspnoeic
$\ldots$ if he experienced chest pain
... if the symptoms lasted longer than 10 minutes
$\ldots$ if chest-pain radiated into arms
... if chest-pain radiated into jaws
Patient was informed that the ED is open $24 / 7$
Patient was told that treatment needs to start immediately
Patient was given a prescription
The prescription was for medication
The prescripiton listed 3 different medications
Name of the new medication was given: Aspirin
Dose of Aspirin: 100 mg
Mode of intake of Aspirin: in the morning
Name of the new medication was given: Beloc
Dose of Beloc: 100 mg
Mode of intake of Beloc: in the morning
Name of the new medication was given: Nitroglycerine
Dose of nitroglycerine: $1-2$ pumps
When to take nitroglycerine: acute complaints
When to take nitroglycerine: chest pain
Patient was told that nitroglycerine will help within 2 to 3 minutes
Physician wished the patient all the best

\section{References}

[1] L.I. Horwitz, J.P. Moriarty, C. Chen, R.L. Fogerty, U.C. Brewster, S. Kanade, et al., Quality of discharge practices and patient understanding at an academic medical center, JAMA Intern. Med. 8093 (2013) 1715-1722, doi:http://dx.doi. org/10.1001/jamainternmed.2013.9318.

[2] A.N. Makaryus, E.A. Friedman, Patients' understanding of their treatment plans and diagnosis at discharge, Mayo Clin. Proc. 80 (2005) 991-994, doi:http://dx. doi.org/10.4065/80.8.991.

[3] L.O. Hansen, R.S. Young, K. Hinami, A. Leung, M.V. Williams, Interventions to reduce 30-day rehospitalization: a systematic review, Ann. Intern. Med. 155 (2011) 520-528, doi:http://dx.doi.org/10.7326/0003-4819-155-8-20111018000008.

[4] K.V. Rhodes, T. Vieth, T. He, A. Miller, D.S. Howes, Resuscitating the physicianpatient relationship: emergency department communication in an academic medical center, Ann. Emerg. Med. 44 (2004) 2-7, doi:http://dx.doi.org/ 10.1016/j.annemergmed.2004.02.035.

[5] S.M. Motamedi, J. Posadas-Calleja, S. Straus, D.W. Bates, D.L. Lorenzetti, B. Baylis, et al., The efficacy of computer-enabled discharge communication interventions: a systematic review, BMJ Qual. Saf. 20 (2011) 403-415, doi: http://dx.doi.org/10.1136/bmjqs.2009.034587.

[6] M.J. Santana, J. Holroyd-Leduc, D.A. Southern, W.W. Flemons, M. O’Beirne, M.D. Hill, et al., A randomised controlled trial assessing the efficacy of an electronic discharge communication tool for preventing death or hospital readmission, BMJ Qual. Saf. 0 (2017) 1-11, doi:http://dx.doi.org/10.1136/bmjqs-2017006635.

[7] S. Kripalani, A.T. Jackson, J.L. Schnipper, E.A. Coleman, Promoting effective transitions of care at hospital discharge: a review of key issues for hospitalists, J. Hosp. Med. 2 (2007) 314-323, doi:http://dx.doi.org/10.1002/jhm.228.

[8] G.A. Miller, The magical number seven, plus or minus two: some limits on our capacity for processing information, Psychol. Rev. 63 (1956) 81-97, doi:http:// dx.doi.org/10.1037/h0043158.

[9] J.J.G. Van Merriënboer, J. Sweller, Cognitive load theory in health professional education: design principles and strategies, Med. Educ. 44 (2010) 85-93, doi: http://dx.doi.org/10.1111/j.1365-2923.2009.03498.x.

[10] K. Wildi, B. Nelles, R. Twerenbold, M. Rubini Giménez, T. Reichlin, H. Singeisen, et al., Safety and efficacy of the $0 \mathrm{~h} / 3 \mathrm{~h}$ protocol for rapid rule out of myocardial infarction, Am. Heart J. 181 (2016) 16-25, doi:http://dx.doi.org/10.1016/j. ahj.2016.07.013. 
[11] J. Hattie, Visible Learning: a Synthesis of over 800 Meta-analyses Relating to Achievement, Routledge, New York, 2013.

[12] G. Brod, M. Werkle-Bergner, Y.L. Shing, The influence of prior knowledge on memory: a developmental cognitive neuroscience perspective, Front. Behav. Neurosci. 7 (2013) 1-13, doi:http://dx.doi.org/10.3389/fnbeh.2013.00139.

[13] W. Langewitz, S. Ackermann, A. Heierle, R. Hertwig, L. Ghanim, R. Bingisser, Improving patient recall of information: harnessing the power of structure, Patient Educ. Couns. 98 (2015) 716-721, doi:http://dx.doi.org/10.1016/j. pec.2015.02.003.

[14] P. Ley, Memory for medical information, Br. J. Clin. Psychol. 18 (1979) 245-255, doi:http://dx.doi.org/10.1111/j.2044-8260.1979.tb00333.x.

[15] S. Ackermann, L. Ghanim, A. Heierle, R. Hertwig, W. Langewitz, R. Mata, et al., Information structuring improves recall of emergency discharge information: a randomized clinical trial, Psychol. Health Med. 8506 (2016) 1-17, doi:http:// dx.doi.org/10.1080/13548506.2016.1198816.

[16] A. Livingston, R.G. Frankiewicz, R.E. Williams, Facilitation of learning and repetition of oral instruction using advanced and post organizers, J. Educ. Psychol. 71 (1979) 701-707, doi:http://dx.doi.org/10.1037/00220663.71.5.701.

[17] J.A. Butz, S.P. Miller, C. Butz, Effect of post-organizers on preservice teachers' content knowledge and understanding of effective teaching behaviors, Teach. Educ. 41 (2005) 1-15, doi:http://dx.doi.org/10.1080/08878730509555368.

[18] D. Nutbeam, The evolving concept of health literacy, Soc. Sci. Med. 67 (2008) 2072-2078, doi:http://dx.doi.org/10.1016/j.socscimed.2008.09.050.

[19] S. Ackermann, A. Heierle, M.-B. Bingisser, R. Hertwig, R. Padiyath, C.H. Nickel, et al., Discharge communication in patients presenting to the emergency department with chest pain: defining the ideal content, Health Commun. 31 (2016) 557-565, doi:http://dx.doi.org/10.1080/10410236.2014.979115.

[20] L. Burla, B. Knierim, J. Barth, K. Liewald, M. Duetz, T. Abel, From text to codings: intercoder reliability assessment in qualitative content analysis, Nurs. Res. 57 (2008) 113-117, doi:http://dx.doi.org/10.1097/01.NNR.0000313482.33917.7d.
[21] R.P.C. Kessels, Patients' memory for medical information, J. R. Soc. Med. 96 (2003) 219-222, doi:http://dx.doi.org/10.1258/jrsm.96.5.219.

[22] B.K. Sanderson, J. Thompson, T.M. Brown, M.J. Tucker, V. Bittner, Assessing patient recall of discharge instructions for acute myocardial infarction, J. Healthc. Qual. 31 (2009) 25-34, doi:http://dx.doi.org/10.1111/j.19451474.2009.00052.x.

[23] J.R. Price, R.A. Mayou, C.M. Bass, R.J. Hames, D. Sprigings, J.S. Birkhead, Developing a rapid access chest pain clinic: qualitative studies of patients' needs and experiences, J. Psychosom. Res. 59 (2005) 237-246, doi:http://dx. doi.org/10.1016/j.jpsychores.2005.04.004.

[24] S. Ackermann, M.B. Bingisser, A. Heierle, W. Langewitz, R. Hertwig, R. Bingisser Discharge communication in the emergency department: physicians underestimate the time needed, Swiss Med. 142 (2012) 1-6, doi:http://dx.doi. org/10.4414/smw.2012.13588.

[25] D. Issacman, K. Purvis, J. Gyuro, Y. Anderson, D. Smith, Standardised instructions: do they improve communication of discharge from the emergency department, Pediatrics 89 (1992) 1204-1207.

[26] B.T. Jolly, J.L. Scott, S.M. Sanford, Simplification of emergency department discharge instructions improves patient comprehension, Ann. Emerg. Med. 26 (1995) 443-446.

[27] J. Jansen, P.N. Butow, J.C.M. Van Weert, S. Van Dulmen, R.J. Devine, T.J. Heeren, et al., Does age really matter? Recall of information presented to newly referred patients with cancer, J. Clin. Oncol. 26 (2008) 5450-5457, doi:http:// dx.doi.org/10.1200/JCO.2007.15.2322.

[28] S.C. Brown, D.C. Park, Theoretical models of cognitive aging and implications for translational research in medicine, Gerontologist 43 (2003) 57-67, doi: http://dx.doi.org/10.1093/geront/43.suppl_1.57.

[29] L. Schwabe, O.T. Wolf, Learning under stress impairs memory formation, Neurobiol. Learn. Mem. 93 (2010) 183-188, doi:http://dx.doi.org/10.1016/j. nlm.2009.09.009. 